\title{
Dynamics of conversion of supercurrents into normal currents, and vice versa
}

\author{
Arne Jacobs and Reiner Kümmel \\ Institut für Theoretische Physik, Universität Würzburg, D-97074 Würzburg, Germany
}

(October 30, 2018)

The generation and destruction of the supercurrent in a superconductor $(S)$ between two resistive normal $(N)$ current leads connected to a current source is computed from the source equation for the supercurrent density. This equation relates the gradient of the pair potential's phase to electron and hole wavepackets that create and destroy Cooper pairs in the $N / S$ interfaces. Total Andreev reflection and supercurrent transmission of electrons and holes are coupled together by the phase rigidity of the non-bosonic Cooper-pair condensate. The calculations are illustrated by snapshots from a computer film.

PACS: 74.25.Fy, 74.80.Dm, 74.80.Fp

\section{INTRODUCTION}

Andreev scattering (AS) of electrons into holes and vice versa by spatial variations of the superconducting pair potential [1], in competition and cooperation with conventional scattering, determines the electronic structure and transport properties of inhomogeneous superconductors. The Tomasch effect in tunnel junctions [2], Josephson currents [3 8], excess currents, and subharmonic gap structures $[12$ in superconducting $(S)$ normal conducting $(N)$-superconducting junctions, as well as the transfer of half of the Magnus force to the core electrons of a moving vortex line $[13$ are due to AS. It is involved in the persistent currents around the AharonovBohm flux in an $N / S$ metal loop [14], and there is AS in $\mathrm{He}^{3}$, too [15]. A wealth of AS phenomena has been discussed recently in [16] and [17].

While the conversion of a normal current into a supercurrent by electron $\rightarrow$ hole scattering in the interface between an $N$ and an $S$ region of semi-infinite lengths has been described before [18, 19, 9], the reverse process has not. Thus, it is the purpose of this paper to analyze the normal-current $\longleftrightarrow$ supercurrent conversion processes in a superconducting layer of finite length $L_{z}$ between two normal current leads. These normal leads are connected to a reservoir ("battery") which acts as the current source in the closed circuit. The extensions of the $N$ and $S$ regions in $x$ - and $y$-directions are $L_{x}$ and $L_{y}$; assuming that they don't exceed the London penetration length one may neglect inhomogeneities of the current density in the superconductor. The metal-vacuum boundaries are treated as rigid walls. By varying $L_{x}$ and $L_{y}$ one can vary the dimensionality of the system. By showing in detail how in any transport experiment involving superconductors electron $\leftrightarrow$ hole scattering brings about normal current $\leftrightarrow$ supercurrent conversion our analysis may also prove useful for the understanding of transport phenomena in quasi-two-dimensional (Q2D) superconducting/semiconducting heterojunctions 2022 and superconducting quantum dots in Q2D channels.

\section{CHARGE CONSERVATION}

AS and the associated formation and destruction of Cooper pairs and supercurrents can be calculated from the Time-dependent Bogoliubov-de Gennes Equations (TdBdGE) 18, 19, 9,23. They describe the evolution of the spinor quasiparticle (q.p.) wavefunction with the electron component $u_{n}(\mathbf{r}, t)$ and the hole component $v_{n}(\mathbf{r}, t)$ under the influence of scalar and vector potentials $V(\mathbf{r}, t)$ and $\mathbf{A}(\mathbf{r}, t)$ in the single-electron Hamiltonian

$$
H_{0}(\mathbf{r}, t)=\frac{1}{2 m}\left[\frac{\hbar}{i} \nabla-e \mathbf{A}(\mathbf{r}, t)\right]^{2}+V(\mathbf{r}, t)-\mu
$$

via the matrix equation

$$
i \hbar \frac{\partial}{\partial t}\left(\begin{array}{l}
u_{n}(\mathbf{r}, t) \\
v_{n}(\mathbf{r}, t)
\end{array}\right)=\check{\mathcal{H}}(\mathbf{r}, t)\left(\begin{array}{l}
u_{n}(\mathbf{r}, t) \\
v_{n}(\mathbf{r}, t)
\end{array}\right) .
$$

Here, the matrix Hamiltonian $\check{\mathcal{H}}(\mathbf{r}, t)$ has $H_{0}(\mathbf{r}, t)$ and $-H_{0}^{*}(\mathbf{r}, t)$ in the diagonal, and the pair potential $\Delta(\mathbf{r}, t)$ and its complex-conjugate in the off-diagonal. The chemical potential $\mu$ in $H_{0}$ is that of the reservoir. We neglect all influences of entropy production associated with current flow on the chemical potential, because the number of degrees of freedom of the reservoir is assumed to be much larger than that of the normal leads and the superconductor. Thus, $\mu$ is constant in space and time [23]. The index $n$ characterizes the stationary q.p. states from which the solutions of eq. (1) evolve after time-dependent scalar and vector potentials have been switched on.

AS is a many-body process. For its analysis it is convenient to consider a non-equilibrium configuration $\left|T_{l \sigma}\right\rangle$ of the many-body system where one quasiparticle state $(l \sigma)$, characterized by a tripel $l$ of quantum numbers and spin $\sigma$, is definitely occupied and all other q.p. states $(n \sigma)$ are occupied according to the equilibrium distribution function $f_{n}$. All interactions that might affect the spin are neglected. Then, it has been shown [18,23] with the help of the TdBdGE (1i) that the expectation values $\left\langle T_{l \sigma}|\rho(\mathbf{r}, t)| T_{l \sigma}\right\rangle$ and $\left\langle T_{l \sigma}|\mathbf{j}(\mathbf{r}, t)| T_{l \sigma}\right\rangle$ of the manybody charge- and current-density operators satisfy the relation 


$$
\begin{gathered}
\frac{\partial}{\partial t}\left\langle T_{l \sigma}|\rho(\mathbf{r}, t)| T_{l \sigma}\right\rangle+\operatorname{div}\left\langle T_{l \sigma}|\mathbf{j}(\mathbf{r}, t)| T_{l \sigma}\right\rangle \\
=-4 \frac{e}{\hbar} \operatorname{Im}\left[\Delta^{*}(\mathbf{r}, t) u_{l}(\mathbf{r}, t) v_{l}^{*}(\mathbf{r}, t)\right]\left(1-f_{l}\right)+\operatorname{div} \mathbf{j}_{s l} .
\end{gathered}
$$

The electron and hole wavefunctions $u_{l}$ and $v_{l}$ satisfy eq. (11), and $\mathbf{j}_{s l}$ is the supercurrent density induced by the momentum and charge transfer from the q.p. in $(l \sigma)$ to the superconducting condensate. All mean-field q.p. states in $\mathbf{j}_{s l}$ and in the selfconsistency equation for $\Delta(\mathbf{r}, t)$ are in a Hilbert space specified to $\left|T_{l \sigma}\right\rangle$. Therefore, their wavefunctions all acquire the same phase shift $S_{l}(\mathbf{r}, t)$ caused by the q.p. in $(l, \sigma)$. This leads to a phase shift $2 S_{l}(\mathbf{r}, t)$ of the pair potential, and $\mathbf{j}_{s l}$ becomes proportional to the gradient of $S_{l}(\mathbf{r}, t)$ times the number $N$ of electrons in the superconductor 18,23]. (Recently the necessity of a phase gradient for charge conservation in $N / S / N$ junctions has again been pointed out by SánchezCañizares and Sols [24].) The requirement that charge is conserved in the many-body system results in the fundamental source equation for the supercurrent density

$$
\operatorname{div}_{s l}=4 \frac{e}{\hbar} \operatorname{Im}\left[\Delta^{*}(\mathbf{r}, t) u_{l}(\mathbf{r}, t) v_{l}^{*}(\mathbf{r}, t)\right]\left(1-f_{l}\right) .
$$

This equation 18, 19, 9,23 has a non-vanishing right-hand side if the $u_{l}$ and $v_{l}$ describe quasiparticles that decay exponentially in the superconductor during total Andreev reflection, because their energies are less than the maximum value of $\Delta$. In this case the source equation yields a finite supercurrent $\mathbf{j}_{s l}$. The phase shift of the pair potential $2 S_{l}$, on the other hand, is essentially given by the (integral of the) r.h.s. of eq. (2), divided by $N \ggg 1$ [18]. Thus, despite of its importance for charge conservation, its numerically tiny value can be neglected in our calculation of solutions of eq. (1).

\section{CURRENT FLOW AND REPRESENTATIVE WAVEPACKETS}

A shifted Fermi sphere (or its equivalent in Q2D and Q1D conductors) represents the current-carrying manybody configurations in the two parts of the normal current leads that are parallel to the $z$-axis and connected to the superconductor. (The direction of current flow in the parts bent towards the reservoir is irrelevant.) These leads, supposed to be much longer than the mean inelastic scattering length, are conductors with resistance [25]. In this non-equilibrium distribution of electrons above the Fermi surface in states with positive momentum in $z$-direction and unoccupied states with negative $z$-momentum below the Fermi surface the current-driving force from the battery is balanced by the frictional forces from the energy-relaxation processes. The quasiparticles in this resistive-state configuration are uncorrelated. Thus, the total current in the closed circuit is the sum of the currents from the individual quasiparticles.
In the following we try to obtain the details of normal current $\longleftrightarrow$ supercurrent conversion by studying the motion of the electrons $(+)$ and holes $(-)$ that are part of the shifted Fermi sphere. Their $z$-momenta are $\hbar k^{ \pm}(E)$, with $k^{ \pm}(E)=\left[k_{z F}^{2} \pm E 2 m / \hbar^{2}\right]^{1 / 2}$. Here, $k_{z F}=$ $\left[k_{F}^{2}-\left(n_{x} \pi / L_{x}\right)^{2}-\left(n_{y} \pi / L_{y}\right)^{2}\right]^{1 / 2}$ is the $z$-component of the Fermi wavenumber $k_{F} \equiv\left[2 m \mu / \hbar^{2}\right]^{1 / 2}$, and $\left(n_{x} \pi / L_{x}\right)$ and $\left(n_{y} \pi / L_{y}\right), n_{x, y}$ integers, are the wavenumbers of the standing waves between the rigid walls that limit the metals in $x$ - and $y$-directions. The energy $E$ of both electrons and holes is positive and measured relative to the surface of the unshifted Fermi sphere at the chemical potential $\mu$. For normal current densities below the critical current densities of conventional superconductors all $E$ are less than the modulus $\Delta$ of the pair potential $\Delta(z) \approx \Delta \cdot \Theta(z) \Theta\left(L_{z}-z\right)$; here $\Theta(z)$ is the Heavyside function which is sufficient to model the spatial variation of the pair potential in the context of current flow [3, 7, 14, 26,27]. Details of the change of $\Delta(z)$ close to the interfaces because of the proximity effect matter little in the integral of eq. (2) that yields the supercurrent.

The motion of wavepackets shows best the dynamics of quantum-mechanical processes. Thus, similar to the use of representative, well-controlled, preformed wavepackets in the calculation of differential cross sections for conventional scattering, we investigate the current dynamics in a closed $N / S / N$ circuit by studying the motion of wavepackets that are representative for the electronic configuration in any transport experiment involving a superconductor connected to a current source by normal conductors. Conventional scattering processes are disregarded because we are only interested in AS in the $N / S$ interfaces. In principle, impurity scattering could be treated with the help of the scattering matrix formulation [27,28]. This is especially convenient for devices that, unlike the ones considered by us, involve only a small number of incoming and outgoing channels. Interface barriers that weaken Andreev scattering have been considered previously [9, 10, 29]; the competition between conventional and Andreev scattering has been discussed in terms of the diagonal and off-diagonal forces associated with broken symmetries [6], and a motion picture of the wavepacket dynamics in such a case can be viewed in the Internet under the address given in the caption of Fig. 1. If conventional scattering is present, the Gaussian spectral function $D(E)$, see below, has to be multiplied by the probability amplitude of transmission. There are only few scattering impurities and no interface barriers present in a Q2D electron gas in a modulation-doped system consisting of an InAs channel between an AlSb substrate and a superconducting Niobium layer that induces a pair potential in the electron gas via the proximity effect [30]. For such an experimental set-up our calculations apply exactly (within the limits of the Andreev approximation). For the general case of any superconductor between any two normal current leads they show the quantum mechanically and electrodynamically 
essential dynamics of AS that rules the charge transport in addition to conventional scattering.

We start our analysis with the initial condition that a normalized electron wavepacket, localized around $z_{0}<0$ in the normal current lead to the left of the superconductor at time $t=0$, travels towards the superconductor. In the center of the wavepacket the energy is $E_{l}<\Delta$. (By varying $E_{l}$ and $k_{z F}$ one can obtain all the low-lying electron excitations that are part of the shifted Fermi sphere in the left normal lead.) For convenience we choose a Gaussian spectral function

$$
D(E)=\frac{a_{z}}{\sqrt{2 \pi}} e^{-\left[k^{+}(E)-k^{+}\left(E_{l}\right)\right]^{2} a_{z}^{2} / 2} e^{-i\left[k^{+}(E)-k^{+}\left(E_{l}\right)\right] z_{0}} ;
$$

the position-uncertainty parameter $a_{z} \ll\left|z_{0}\right|$ is chosen so large that the related energy spread of the wavepacket, $\delta_{E}=\hbar^{2} k^{+}\left(E_{l}\right) / m a_{z}$, is less than $\Delta-E_{l}$. Solutions of eq. (1) are calculated neglecting $V$ and $\mathbf{A}$ and approximating $\Delta(\mathbf{r}, t)$ by the real $\Delta(z)$, thereby disregarding repercussions of the quasiparticle-induced supercurrent on the q.p. 13] and on itself. (As discussed above, the phase shift of the pair potential due to one Andreev reflection process is negligibly small.) These solutions are multiplied by the spectral function $D(E)$, integrated over all energies, and matched at the left $N / S$ interface, i.e. $z=0$, in the usual Andreev approximation where terms of the order of $\Delta / \mu$ are neglected outside the exponentials. Energy-dependent functions are Taylor expanded around $E_{l}$ up to first order in $\left(E-E_{l}\right)$. This affects the amplitude of the Andreev-reflection probability

$$
\gamma(E) \equiv e^{-i \arccos E / \Delta} \approx \gamma\left(E_{l}\right) e^{(i / \hbar)\left(E-E_{l}\right) \tau_{l}}
$$

and $k^{ \pm}(E)=\left[k_{z F}^{2} \pm E 2 m / \hbar^{2}\right]^{1 / 2} \approx k_{l}^{ \pm} \pm\left(E-E_{l}\right) / \hbar v_{l}^{ \pm}$; $\tau_{l}=\hbar\left[\Delta^{2}-E_{l}^{2}\right]^{-1 / 2}$ is the time for one electron $\rightarrow$ hole-scattering event and the associated formation of a Cooper pair, see eqs. (3, 俚), and

$$
k_{l}^{ \pm} \equiv k^{ \pm}\left(E_{l}\right), \quad v_{l}^{ \pm} \equiv \hbar k_{l}^{ \pm} / m .
$$

The resulting electron and hole wavepackets $u_{N L}(\mathbf{r}, t)$ and $v_{N L}(\mathbf{r}, t)$ in the left normal current lead, $z<0$, and the exponentially decaying solutions $u_{S L}(\mathbf{r}, t)$ and $v_{S L}(\mathbf{r}, t)$ in $z>0$, that contribute to the source equation (2) essentially in the left half of the superconductor, turn out to be

$$
\begin{aligned}
& u_{N L}=w_{l} e^{i k_{l}^{+} z} e^{-\left[z_{0}-z+v_{l}^{+} t\right]^{2} / 2 a_{z}^{2}}, \\
& v_{N L}=w_{l} \gamma\left(E_{l}\right) e^{i k_{l}^{-} z} e^{-\left[z_{0}+\left(v_{l}^{+} / v_{l}^{-}\right) z+v_{l}^{+}\left(t-\tau_{l}\right)\right]^{2} / 2 a_{z}^{2}}, \\
& u_{S L}=w_{l} e^{i k_{z F} z} e^{-\kappa_{l} z} e^{-\left[z_{0}+v_{l}^{+} t\right]^{2} / 2 a_{z}^{2}} \\
& v_{S L}=w_{l} \gamma\left(E_{l}\right) e^{i k_{z F} z} e^{-\kappa_{l} z} e^{-\left[z_{0}+v_{l}^{+}\left(t-\tau_{l}\right)\right]^{2} / 2 a_{z}^{2}},
\end{aligned}
$$

where

$$
w_{l} \equiv \frac{2}{\left(L_{x} L_{y} a_{z} \sqrt{\pi}\right)^{1 / 2}} \sin \left(\frac{n_{x} \pi}{L_{x}} x\right) \sin \left(\frac{n_{y} \pi}{L_{y}} y\right) e^{-i E_{l} t / \hbar},
$$

and $\kappa_{l} \equiv\left(\Delta^{2}-E_{l}^{2}\right)^{1 / 2} / \hbar v_{z F}=1 / \tau_{l} v_{z F}$, with $v_{z F} \equiv$ $\hbar k_{z F} / m$. (For the sake of simplicity the complex wavenumbers in $u_{S L}$ and $v_{S L}$ have not been Taylor expanded in $\left(E-E_{l}\right)$ but rather taken at $E_{l}$ right away.)

Identifying the wavefunctions $u_{S L}$ and $v_{S L}$ from eqs. (5) and (6) with the $u_{l}$ and $v_{l}$ of the source equation (2) and integrating that equation from $z=0$ to $z$ yields the density of the supercurrent in $z$-direction, induced in the left half of the superconductor by AS of the electron wavepacket $u_{N L}$ into the hole wavepacket $v_{N L}$, as

$$
\begin{aligned}
\mathbf{j}_{s l, L}= & \mathbf{e}_{z}\left(2 e v_{z F}\right)\left|w_{l}\right|^{2}\left[1-e^{-2 \kappa_{l} z}\right] \\
& \times e^{-\left\{\left[z_{0}+v_{l}^{+}\left(t-\tau_{l} / 2\right)\right]^{2}+\left(\tau_{l} v_{l}^{+} / 2\right)^{2}\right\} / a_{z}^{2}}\left(1-f_{l}\right) .
\end{aligned}
$$

Here we have assumed that $L_{z} \gg 1 / \kappa_{l}$. In the opposite case one would have to add a second source term on the r.h.s. of eq. (2). This term would contain the contribution from the wavepacket solution $u_{S R}(\mathbf{r}, t), v_{S R}(\mathbf{r}, t)$ of eq. (11) for $0<z<L_{z}$ that matches to the currentcarrying q.p. wavepackets in the right normal current lead at $z=L_{z}$ and decays exponentially with increasing distance from $L_{z}$. However, in our case of large $L_{z}$ the $u_{S R}(\mathbf{r}, t)$ and $v_{S R}(\mathbf{r}, t)$ give only rise to the supercurrent $\mathbf{j}_{s l, R}$ in the right half of the superconductor. The complex amplitudes of the solutions of the TdBdGE in the energy integrals that form the wavepackets $u_{S R}(\mathbf{r}, t)$ and $v_{S R}(\mathbf{r}, t)$ are uniquely determined by the requirement that the supercurrent densities $\mathbf{j}_{s l, L}$ and $\mathbf{j}_{s l, R}$, computed from $u_{S L} v_{S L}^{*}$ and $u_{S R} v_{S R}^{*}$, join smoothly at all times somewhere within the superconductor. Since the phases of the Fermi-liquid quasiparticles in the left and right normal current leads are at random, only the current densities, not the wavefunctions, must join smoothly. (If, on the other hand, the superconductor were only a thin slab with $L_{z} \ll 1 / \kappa_{l}$, there would be a finite probability that the q.p. do not suffer AS and induce a supercurrent but rather carry their phases through the pair-potential wall in a tunneling process.) The matching point turns out to be $L_{z} / 2$, and the evanescent wavepackets result to be

$$
\begin{aligned}
& u_{S R}=w_{l} e^{i k_{z F} z} e^{-\kappa_{l}\left(L_{z}-z\right)} e^{-\left[z_{0}+v_{l}^{+}\left(t-\tau_{l}\right)\right]^{2} / 2 a_{z}^{2}}, \\
& v_{S R}=w_{l} \gamma\left(E_{l}\right)^{-1} e^{i k_{z F} z} e^{-\kappa_{l}\left(L_{z}-z\right)} e^{-\left[z_{0}+v_{l}^{+} t\right]^{2} / 2 a_{z}^{2}} .
\end{aligned}
$$

More details are given in the Appendix.

The supercurrent density $\mathbf{j}_{s l, R}$, obtained by integrating eq. (2) from $L_{z}$ to $z$, with $u_{S R} v_{S R}^{*}$ in the place of $u_{l} v_{l}^{*}$, has the same form as $\mathbf{j}_{s l, L}$ of eq. (7) except that $\exp \left[-2 \kappa_{l} z\right]$ is being replaced by $\exp \left[-2 \kappa_{l}\left(L_{z}-z\right)\right]$. Finally, the wavepacket solutions $u_{N R}(\mathbf{r}, t), v_{N R}(\mathbf{r}, t)$ of eq. (11) in the right normal current lead, $z>L_{z}$, that match to the $u_{S R}(\mathbf{r}, t), v_{S R}(\mathbf{r}, t)$ at the right interface in $z=L_{z}$, become

$$
\begin{aligned}
u_{N R}= & w_{l} e^{i k_{z F} L_{z}} e^{i k_{l}^{+}\left(z-L_{z}\right)} \\
& \times e^{-\left[z_{0}+L_{z}-z+v_{l}^{+}\left(t-\tau_{l}\right)\right]^{2} / 2 a_{z}^{2}}, \\
v_{N R}= & w_{l} \gamma\left(E_{l}\right)^{-1} e^{i k_{z F} L_{z}} e^{i k_{l}^{-}\left(z-L_{z}\right)} \\
& \times e^{-\left[z_{0}+\left(v_{l}^{+} / v_{l}^{-}\right)\left(z-L_{z}\right)+v_{l}^{+} t\right]^{2} / 2 a_{z}^{2}} .
\end{aligned}
$$


Note that these wavepackets are the result of only one initial condition, namely "electron wavepacket incident from the left". This initial condition, the matching of the wavepackets at the interfaces, the requirement of charge conservation as expressed by eq. (2), and the smooth joining of the supercurrent densities $\mathbf{j}_{s l, L}$ and $\mathbf{j}_{s l, R}$ determine unequivocally the wavepackets in the right normal current lead (apart from an irrelevant, constant phase factor that has deliberately been set equal to unity).

\section{ANDREEV SCATTERING AND COOPER PAIR FORMATION}

Comparison of the $u_{N L}, v_{N L}$ from eqs. (3, th) with the $u_{N R}, v_{N R}$ from eqs. (10,11) shows that the center of the electron wavepacket $u_{N L}$, propagating to the right with velocity $v_{l}^{+}$in the left normal current lead, and the center of the hole wavepacket $v_{N R}$, propagating to the left with velocity $v_{l}^{-}$in the right normal current lead, hit the left and right interfaces at $z=0$ and $z=L_{z}$ (with different phases) at the same time $t_{0}=-z_{0} / v_{l}^{+}$, while the hole wavepacket $v_{N L}$, propagating to the left in $z<0$ with $v_{l}^{-}$, and the electron wavepacket $u_{N R}$, propagating to the right in $z>L_{z}$, are retarded by the time $\tau_{l}$ with respect to the incident wavepackets. The holes moving to the left transport positive momentum and negative charge to the right, just as the electrons of opposite group velocity do. The supercurrent density $\mathbf{j}_{s l}$ spreads "instantaneously" throughout the superconductor (the velocity of light not exceeding, of course) and couples directly electron $\rightarrow$ hole scattering in the left to hole $\rightarrow$ electron scattering in the right interface.

This "instantaneous" coupling may seem to be surprising, but only at a first look. Giving it a second thought one sees that our result confirms by explicit wavepacket calculation, and for the first time, as far as we know, what one has concluded before intuitively and from steady state calculations: hole $\rightarrow$ electron scattering destroys and electron $\rightarrow$ hole scattering creates supercurrents, whenever currents flow through $S / N$ and $N / S$ interfaces, e.g. in vortex lines [13]. Both scattering processes must occur simultaneously even at far-apart interfaces because of charge conservation in closed circuits and as a direct consequence of the very essence of BCS superconductivity: Cooper pairs are not bosons, despite of what one can read frequently, because within the volume of one Cooper pair of a conventional superconductor there are about one million of mass centers of other Cooper pairs, their creation and destruction operators do not satisfy bosonic commutation relations, and their condensate wavefunction is antisymmetric [32]. Therefore, the Cooper pair formed by total Andreev reflection in one interface cannot exist outside but only within the condensate to which the momentum $2 \hbar k_{z F}$ and the charge of the two electrons of opposite spin, that have entered the superconductor, must be transferred. Because of the "phase rigidity of the electron pair fluid" [34], so typical for off-diagonal long range order, this charge and momentum transfer, and the related phase shift, manifest themselves immediately in a current flow out of the other interface. Thus, no charges can accumulate in the superconductor according to its capacitance (as charges from quasiparticles with energies $E>\Delta$ may do), and the reservoir has to emit holes into and receive electrons from the right normal lead at the same rate at which it emits electrons into and receives holes from the left current lead. This is illustrated by the snapshots from a computer film in Figure 1. The Andreev-reflected wavepackets may be considered as supercurrent-transmitted wavepackets as well. Together with the incident wavepackets they represent any of the low lying single-particle excitations with energies $E_{l}<\Delta$ in the current-carrying, resistive parts of the circuit.

Considering the supercurrent contribution from our representative quasiparticle-wavepacket configuration we note that its spatial maximum is at $z=L_{z} / 2$, and its maximum in time occurs at $t=t_{0}+\tau_{l} / 2$. From the equations for $\mathbf{j}_{s l}, u_{S L, R}$ and $v_{S L, R}$ one sees that the quasiparticle current density

$$
\mathbf{j}_{Q P l} \equiv \frac{e}{m} \operatorname{Re}\left[u_{l}^{*} \frac{\hbar}{i} \nabla u_{l}-v_{l} \frac{\hbar}{i} \nabla v_{l}^{*}\right]\left(1-f_{l}\right)
$$

changes into the supercurrent density $\mathbf{j}_{s l}$ and vice versa within a distance $1 / \kappa_{l}=v_{z F} \tau_{l}$ from the interfaces.

In conclusion, the combinations of single-particle excitations (wavepackets) and collective modes (supercurrents), connected by AS as shown in Fig. 1, are the consequence of the phase rigidity of the Cooper-pair condensate and the adjustment of the current configuration to charge conservation in the $N / S$ and $S / N$ interfaces. They are the current-carrying elementary excitations in closed $N / S / N$ circuits.

\section{OUTLOOK}

The supercurrent, carried by the condensate in the $S$ layer, involves only states with $\left|E_{n}\right| \geq \Delta$. It continues the current in the normal current leads where all electrons and holes have energies $E_{l}<\Delta$. If the total current density exceeds its critical value, i.e. if the center of the Fermi sphere in the normal current leads is shifted by more than $\hbar q_{c S}=\Delta m / \hbar k_{F}$, depairing sets in, and when superconductivity has broken down, the uncorrelated normal-state configuration reigns everywhere in the circuit. If, on the other hand, the single $S$ layer is replaced by a mesoscopic $S N S$ junction, the many-body configuration in the central $N$ layer is a phase-coherent one and thus different from the uncorrelated configurations in the normal current leads. In an $N / S N S / N$ circuit the $S N S$ junction acts as a gapless superconductor 沺. It can carry a dissipation-free Josephson current through the central $N$ layer via phasecoherent q.p. states with $|E|<\Delta$ and $|E| \geq \Delta[$ [6]. This current converts as a whole into the total supercurrent of the $S$ layers, and 
vice versa, whereas, according to eq. (2), each uncorrelated q.p. from the normal current leads individually induces its proper fraction of the total supercurrent. If the total current density exceeds the critical Josephsoncurrent density at a Fermi-sphere shift of $\hbar q_{c S N S} \approx \hbar / d$, where $d$ is the length of the central $N$ layer 5 , a voltage drop appears across the $S N S$ junction. There are different models 10 12,33, 8 for $S N S$ junctions with voltage drops. They differ with respect to the implicit assumptions about the rate and energy range of q.p. creation in the central $N$ layer by supercurrent $\longrightarrow$ quasiparticlecurrent conversion. The question of how this rate and range depend upon the weakening of phase coherence in the $S N S$ junction by energy exchange between quasiparticles and electric fields, phonons, and thermal fluctuations like Nyquist-Johnson noise [35] is presently investigated.

\section{APPENDIX: MATCHING OF SUPERCUR- RENTS}

The supercurrent density in the right part of the superconductor, $j_{s l, R}$, results from the evanescent wavepackets $u_{S R}, v_{S R}$. These are built up from the stationary solutions of eq. (11) that decay exponentially within the superconductor with increasing distance from the right interface at $z=L_{z}$. The free amplitudes $\Omega(E)$ of each of these solutions are determined by demanding that $j_{s l, R}$ joins smoothly with the supercurrent density $j_{s l, L}$ from the left part of the superconductor at all times somewhere within the superconductor. Once the amplitudes $\Omega(E)$ are known, the electron- and hole-wavepackets in the right normal current lead are unequivocally determined by eq. (1) and the matching conditions for the wavepackets in $z=L_{z}$.

The remainder of this appendix just shows how the amplitudes $\Omega(E)$ are calculated.

In the right part of the superconductor, with the Gaussian spectral function $D(E)$ and those solutions of eq. (11) that increase as $z<L_{z}$ approaches $L_{z}$, we obtain

$$
\begin{aligned}
u_{S R}= & \sin \left(\frac{n_{x} \pi}{L_{x}} x\right) \sin \left(\frac{n_{y} \pi}{L_{y}} y\right) \frac{1}{\delta_{E} \sqrt{2 \pi}} e^{-i E_{l} t / \hbar} e^{i k_{z F} z} e^{-\kappa_{l}\left(L_{z}-z\right)} \\
& \times \int_{0}^{\infty} \mathrm{d} E e^{-\left[\left(a_{z} / \hbar v_{l}^{+}\right)^{2}\left(E-E_{l}\right)^{2} / 2+i\left(z_{0} / \hbar v_{l}^{+}+t / \hbar\right)\left(E-E_{l}\right)\right]} \Omega(E), \\
v_{S R}= & \sin \left(\frac{n_{x} \pi}{L_{x}} x\right) \sin \left(\frac{n_{y} \pi}{L_{y}} y\right) \frac{1}{\delta_{E} \sqrt{2 \pi}} e^{-i E_{l} t / \hbar} e^{i k_{z F} z} e^{-\kappa_{l}\left(L_{z}-z\right)} \gamma\left(E_{l}\right)^{-1} \\
& \times \int_{0}^{\infty} \mathrm{d} E e^{-\left[\left(a_{z} / \hbar v_{l}^{+}\right)^{2}\left(E-E_{l}\right)^{2} / 2+i\left(z_{0} / \hbar v_{l}^{+}+\left(t+\tau_{l}\right) / \hbar\right)\left(E-E_{l}\right)\right]} \Omega(E) .
\end{aligned}
$$

(The term $\delta_{E}=\hbar^{2} k^{+}\left(E_{l}\right) / m a_{z}$ in the denominator, which results from replacing the wavepacket integration over $k^{+}$by one over $E$, drops out after the evaluation of the integrals.)

Let $z^{\prime}$ be the point where the supercurrent densities $\mathbf{j}_{s l, L}$ and $\mathbf{j}_{s l, R}$ join smoothly. Then the integrals of the source equation (2) must satisfy 


$$
\begin{aligned}
\left.\mathbf{j}_{s l, L}\right|_{z^{\prime}} & =\mathbf{e}_{z} \int_{0}^{z^{\prime}} \mathrm{d} z 4 \frac{e}{\hbar} \operatorname{Im}\left(\Delta^{*} u_{S L} v_{S L}^{*}\right)\left(1-f_{l}\right) \\
& \stackrel{!}{=}-\mathbf{e}_{z} \int_{z^{\prime}}^{L_{z}} \mathrm{~d} z 4 \frac{e}{\hbar} \operatorname{Im}\left(\Delta^{*} u_{S R} v_{S R}^{*}\right)\left(1-f_{l}\right)=\left.\mathbf{j}_{s l, R}\right|_{z^{\prime}}
\end{aligned}
$$

$$
\omega_{1}=\frac{2}{\left(L_{x} L_{y} a_{z} \sqrt{\pi}\right)^{1 / 2}}
$$

For convenience we write the complex $\Omega(E)$ as the product of two factors, one of them being energydependent:

$$
\Omega(E) \equiv \omega_{1} \omega_{2}(E)
$$

Now we insert the $u_{S R}$ and $v_{S R}$ of eqs. (A1) and (A2) into the supercurrent density $\left.\mathbf{j}_{s l, R}\right|_{z^{\prime}}$ of eq. (A3) and, similarly, $\left.\mathbf{j}_{s l, L}\right|_{z^{\prime}}$ is expressed by the solutions $u_{S L}$ and $v_{S L}$ in the same form, i.e. without evaluating the energy integrals:

$$
\begin{aligned}
\left.\mathbf{j}_{s l, L}\right|_{z^{\prime}}= & \mathbf{e}_{z} 4 \frac{e}{\hbar} \Delta \sin ^{2}\left(\frac{n_{x} \pi}{L_{x}} x\right) \sin ^{2}\left(\frac{n_{y} \pi}{L_{y}} y\right) \frac{1}{2 \pi \delta_{E}^{2}} \frac{4}{L_{x} L_{y} a_{z} \sqrt{\pi}} \\
& \times \operatorname{Im}\left\{\gamma\left(E_{l}\right)^{*}\left[\int_{0}^{\infty} \mathrm{d} E e^{-\left[\left(a_{z} / \hbar v_{l}^{+}\right)^{2}\left(E-E_{l}\right)^{2} / 2+i\left(z_{0} / \hbar v_{l}^{+}+t / \hbar\right)\left(E-E_{l}\right)\right]}\right]\right. \\
& \left.\times\left[\int_{0}^{\infty} \mathrm{d} E e^{-\left[\left(a_{z} / \hbar v_{l}^{+}\right)^{2}\left(E-E_{l}\right)^{2} / 2+i\left(z_{0} / \hbar v_{l}^{+}+\left(t-\tau_{l}\right) / \hbar\right)\left(E-E_{l}\right)\right]}\right]^{*}\right\} \\
& \times \int_{0}^{z^{\prime}} \mathrm{d} z e^{-2 \kappa_{l} z}\left(1-f_{l}\right), \\
\left.\mathbf{j}_{s l, R}\right|_{z^{\prime}}= & -\mathbf{e}_{z} 4 \frac{e}{\hbar} \Delta \sin ^{2}\left(\frac{n_{x} \pi}{L_{x}} x\right) \sin ^{2}\left(\frac{n_{y} \pi}{L_{y}} y\right) \frac{1}{2 \pi \delta_{E}^{2}}\left|\omega_{1}\right|^{2} \\
& \times \operatorname{Im}\left\{\gamma\left(E_{l}\right)^{-1}\right]^{*}\left[\int_{0}^{\infty} \mathrm{d} E e^{-\left[\left(a_{z} / \hbar v_{l}^{+}\right)^{2}\left(E-E_{l}\right)^{2} / 2+i\left(z_{0} / \hbar v_{l}^{+}+t / \hbar\right)\left(E-E_{l}\right)\right]} \omega_{2}(E)\right] \\
& \times\left[\int_{0}^{\infty} \mathrm{d} E e^{-\left[\left(a_{z} / \hbar v_{l}^{+}\right)^{2}\left(E-E_{l}\right)^{2} / 2+i\left(z_{0} / \hbar v_{l}^{+}+\left(t+\tau_{l}\right) / \hbar\right)\left(E-E_{l}\right)\right]} \omega_{2}(E)\right] \\
& \times \int_{z^{\prime}}^{L_{z}} \mathrm{~d} z e^{-2 \kappa_{l}\left(L_{z}-z\right)}\left(1-f_{l}\right) .
\end{aligned}
$$

Note that

$$
\operatorname{Im}\left\{\left[\gamma\left(E_{l}\right)^{-1}\right]^{*}\right\}=-\operatorname{Im}\left\{\gamma\left(E_{l}\right)^{*}\right\} .
$$

We demand that the integrals over $z$ are equal at $z^{\prime}$ :

$$
\int_{0}^{z^{\prime}} \mathrm{d} z e^{-2 \kappa_{l} z}=\frac{1}{2 \kappa_{l}}\left(1-e^{-2 \kappa_{l} z^{\prime}}\right) \stackrel{!}{=} \frac{e^{-2 \kappa_{l} L_{z}}}{2 \kappa_{l}}\left(e^{2 \kappa_{l} L_{z}}-e^{2 \kappa_{l} z^{\prime}}\right)=\int_{z^{\prime}}^{L_{z}} \mathrm{~d} z e^{-2 \kappa_{l}\left(L_{z}-z\right)} .
$$

This equation is satisfied by

$$
z^{\prime}=\frac{1}{2} L_{z}
$$

The energy integrals in eqs. (A5) and A6 are real. By comparing them, i.e. the first one in eq. (A5) with the second one in eq. (A6), or the second one in (A5) with the first one in eq. (A6), we find

$$
\omega_{2}(E)=e^{i\left(E-E_{l}\right) \tau_{l} / \hbar}
$$


[1] A. Andreev, Sov. Phys. JETP 19, 1228 (1964), [Zh. Eksp. Teor. Fiz. 46, 1823 (1964)]; Sov. Phys. JETP 22, 455 (1966), [Zh. Eksp. Teor. Fiz. 49, 655 (1965)].

[2] W. McMillan and P. W. Anderson, Phys. Rev. Lett. 16, 85 (1966).

[3] I. Kulik, Sov. Phys. JETP 30, 944 (1970), [Zh. Eksp. Teor. Fiz. 57, 1745 (1969)].

[4] C. Ishii, Progr. Theor. Physics 44, 1525 (1970).

[5] J. Bardeen and J. Johnson, Phys. Rev. B 5, 72 (1972).

[6] U. Gunsenheimer, U. Schüssler, and R. Kümmel, Phys. Rev. B 49, 6111 (1994), and references therein.

[7] H. Kroemer, in [16], pp. 877 - 889

[8] A. Jacobs, R. Kümmel, and H. Plehn, in [16], pp. 669 681.

[9] G. Blonder, M. Tinkham, and T. Klapwijk, Phys. Rev. B 25, 4515 (1982).

[10] M. Octavio, M. Tinkham, G. Blonder, and T. Klapwijk, Phys. Rev. B 27, 6739 (1983).

[11] R. Kümmel, U. Gunsenheimer, and R. Nicolsky, Phys. Rev. B 42, 3992 (1990).

[12] U. Gunsenheimer and A. Zaikin, Phys. Rev. B 50, 6317 (1994).

[13] S. Hofmann and R. Kümmel, Phys. Rev. Lett. 70, 1319 (1993), Phys. Rev. B 57, 7904 (1998).

[14] M. Büttiker and T. Klapwijk, Phys. Rev. B 33, 5114 (1986).

[15] N. Schopohl and D. Waxman, Phys. Rev. Lett. 63, 1696 (1989).

[16] P. Bagwell (Guest Editor), Superlattices and Microstructures, No. 5/6, 25, (1999).

[17] Physics and Applications of Mesoscopic Josephson Junctions, edited by H. Ohta and C. Ishii (The Physical Society of Japan, Tokyo, 1999).

[18] R. Kümmel, Z. Physik 218, 472 (1969).

[19] W. N. Matthews Jr., Phys. Stat. Sol. (b) 90, 327 (1978).

[20] J. Nitta, T. Akazaki, and H. Takayanagi, Phys. Rev. B 49, 3659 (1994)

[21] H. Kroemer et al., Physica B 203, 298 (1994).

[22] G. Bastian et al., Phys. Rev. Lett. 81, 1686 (1998).

[23] R. Kümmel, in 17], pp. 19 - 37.

[24] J. Sánchez-Cañizares and F. Sols, Physica B 252, 304 (1998).

[25] H. Pothier et al., Phys. Rev. Lett. 79, 3490 (1997), analyze the relaxation mechanisms in a resistive conductor at $25 \mathrm{mK}$.

[26] D. V. Averin, Phys. Rev. Lett. 82, 3685 (1999).

[27] C. W. J. Beenakker, Rev. Mod. Phys. 69, 731 (1997).

[28] G. Johansson, G. Wendin, K. N. Bratus, V. S. Shumeiko, in [16], pp. $905-914$.

[29] U. Schüssler, R. Kümmel, Phys. Rev. B 47, 2754 (1993).

[30] Jacobs et al. [8] calculated the proximity-induced pair potential in a narrow InAs channel at $T=2.2 \mathrm{~K}$ from the self-consistency equation in the quasi-classical Green's functions formalism. They assumed various critical temperatures $T_{C N}$ below which InAs would become super- conducting because of a tiny, but non-zero, attractive electron-phonon interaction. They found that even at a $T_{C N}$ of only one thousandth of the critical temperature of Niobium one has a pair potential which is about $10 \%$ of the $\mathrm{Nb}$ pair potential. Alternatively, assuming a vanishing electron-phonon coupling constant, Volkov et al. 31. calculated a small gap in the excitation spectrum of a Q2D electron gas in an InAs channel below two separated $\mathrm{Nb}$ contacts. Multiplying the gap by a phase they considered it as the effective order parameter in the InAs below the $\mathrm{Nb}$ contacts, relevant for Andreev scattering and Josephson currents.

[31] A. F. Volkov, P.H.C. Magnée, B.J. van Wees, and T. M. Klapwijk, Physica C 242, 261 (1995).

[32] J. R. Schrieffer, Theory of Superconductivity (Benjamin, N.Y., 1964), pp. 32-42.

[33] D. Averin and A. Bardas, Phys. Rev. Lett. 75, 1831 (1995).

[34] P. W. Anderson, Basic Notions of Condensed Matter Physics (Benjamin/Cummings, Menlo Park, Cal., 1984), Chapter 2: Broken Symmetry.

[35] V. Niño C. and R. Kümmel, Phys. Rev. B 29, 3957 (1984). 

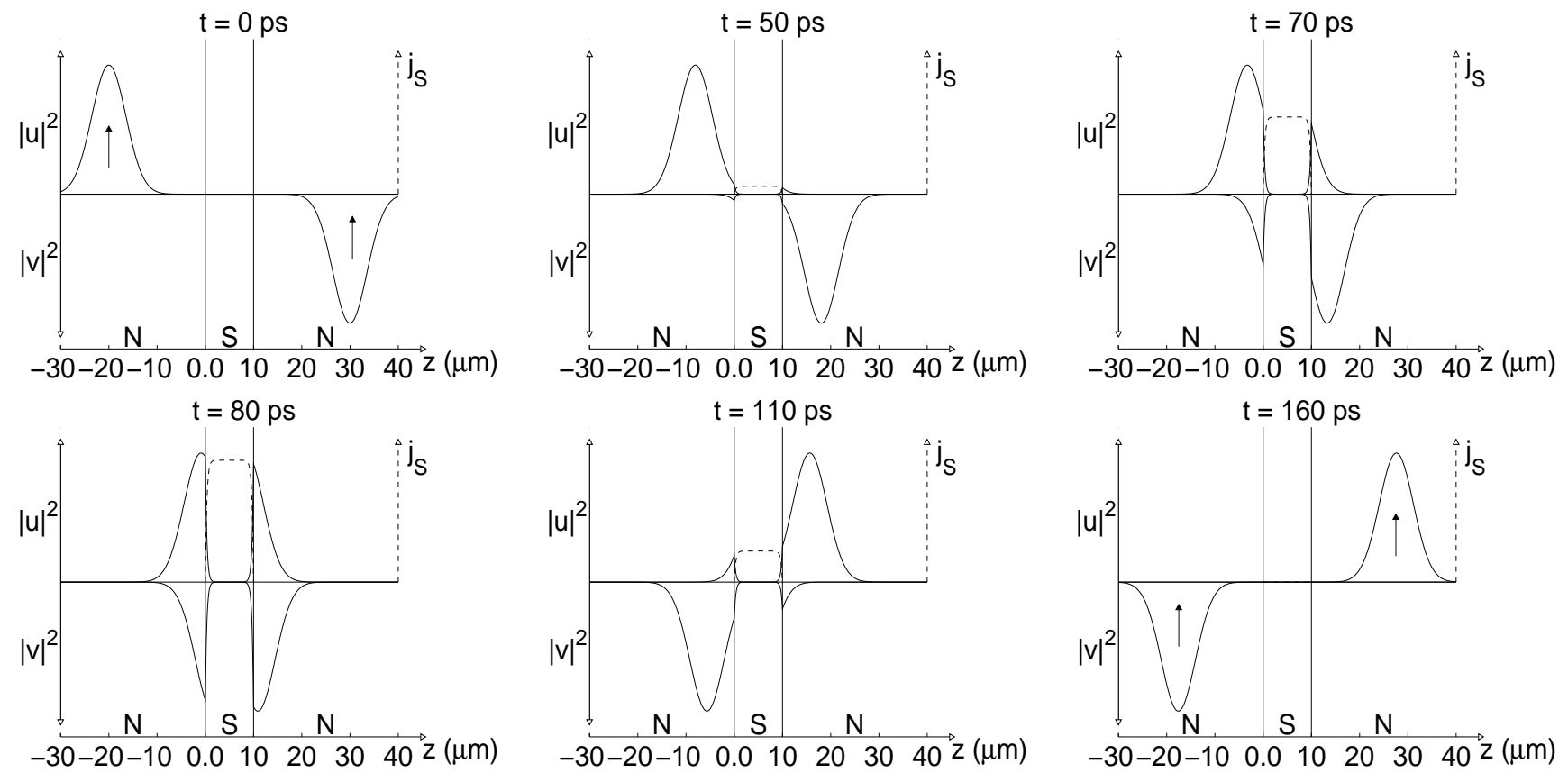

FIG. 1. Propagation and Andreev scattering of the probability densities $|u|^{2}$ and $|v|^{2}$ of a representative spin-up electron- and hole-wavepacket configuration (solid lines), and the induced supercurrrent density $\mathbf{j}_{s}$ (dashed line), in a current-carrying closed $N / S / N$ circuit. For the sake of clear graphical representation the initial conditions for the electron wavepacket, incident from the left, have been chosen as: energy in the center $E_{l}=\Delta / 2=0.15 \mathrm{meV}$, spatial spread $a_{z}=5 \mu \mathrm{m}$, and $k_{z F}=0.9 k_{F}=2.06 \mathrm{~nm}^{-1}$. Via charge conservation by supercurrent induction these conditions determine the parameters and the motion of the resulting hole wavepacket incident from the right; the retardation time for $\mathrm{AS}, \tau_{l}=\hbar /\left[\Delta^{2}-E_{l}^{2}\right]^{1 / 2}$, is about two picoseconds (ps). More computer films on electron $\rightarrow$ hole and electron $\rightarrow$ electron scattering in one $N / S$ interface, also for energies above the gap, can be viewed in the Internet under http://theorie.physik.uni-wuerzburg.de/TP1/kuemmel/films/filmse.htm. 